\section{Citrat im Urin}

W. G. Guder

München, Deutschland

Englischer Begriff citrate in urine; citric acid in urine

Definition Erfassung von Zitronensäure und ihren Salzen im 24-Stunden-Urin.

Struktur $\mathrm{C}_{6} \mathrm{H}_{8} \mathrm{O}_{7}$ (Citronensäure).

Molmasse 192,1 g (als Säure)

Synthese - Verteilung - Abbau - Elimination Citrate und Zitronensäure aus der Nahrung werden im Duodenum resorbiert und in der Niere durch Filtration und Metabolismus im proximalen Tubulus eliminiert. Urin-Citrat kommt im Wesentlichen aus dem sekretorischen und metabolischen Citrat des distalen Tubulus.

Halbwertszeit Im Blut von kurzer Halbwertszeit (wenige Minuten), da glomerulär frei filtrierbar und mit Resorption im proximalen Tubulus eliminiert.

Funktion - Pathophysiologie Distal tubuläre Citratsekretion ist wesentliche Ursache für Citratelimination im Urin. Sie wird gesteigert durch diätetisches Ansäuern des Urins. Da Citrat wesentlicher Faktor für die Löslichkeit von Calcium im Urin ist, wird die Citratausscheidung als Teil des > Steinmetaphylaxe-Programms zur Quantifizierung empfohlen.

Untersuchungsmaterial - Entnahmebedingungen 24Stunden-Sammelurin, instabil im Nativurin, Ansäuern auf $\mathrm{pH}<2$ empfohlen.
Analytik Enzymatisch mit Citratlyase.

Konventionelle Einheit $\mathrm{mg} / 24 \mathrm{~h}$.

Internationale Einheit $\mathrm{mmol} / 24 \mathrm{~h}$.

Umrechnungsfaktor zw. konv. u. int. Einheit Konv. $\times 0,0052=$ intern. bzw. intern. $\times 191,1=$ konvent.

Referenzbereich - Frauen $2-4 \quad \mathrm{mmol} / 24 \quad \mathrm{~h}$ (382-764 mg/24 h).

Referenzbereich - Männer $2-4 \quad \mathrm{mmol} / 24 \quad \mathrm{~h}$ (382-764 mg/24 h).

Referenzbereich - $\begin{array}{llll}\text { Kinder } & 2-4 & \mathrm{mmol} / 24 & \mathrm{~h}\end{array}$ (382-764 mg/24 h).

Indikation Teil des Untersuchungsprogramms von Steinträgern.

Interpretation Ziel der Therapie ist eine Citratausscheidung $>2,5 \mathrm{mmoL} / 24 \mathrm{~h}(>481 \mathrm{mg} / 24 \mathrm{~h})$.

Diagnostische Wertigkeit Da die Citratausscheidung von vielen diätetischen und renalen Faktoren abhängt, ist seine Messung nicht von der gleichen Bedeutung wie die der steinbildenden Substanzen (Oxalat, Calcium, Harnsäure).

\section{Literatur}

Hesse A, Jahnen A, Klocke K et al (1994) Nachsorge bei Harnsteinpatienten. Gustav-Fischer-Verlag, Jena Stuttgart 